\title{
La influencia de Albert Einstein y la Física Cuántica en la narrativa de Kurt Vonnegut
}

\author{
(The influence of Albert Einstein and Quantum Physics in Kurt \\ Vonnegut's narrative)
}

\author{
JAVIER MARTín PÁRRAGA \\ (Grupo de Investigación HUM682)
}

Fecha de recepción: 28 de febrero de 2010
Fecha de aceptación: 30 de marzo de 2010

Resumen: En el presente trabajo se analiza la profunda influencia que las teorías científicas de Albert Einstein y alguno de sus discípulos y colaboradores más cercanos ejerce en la narrativa del novelista norteamericano de origen alemán Kurt Vonnegut (1922-2007). Para llevar a cabo el objetivo propuesto, en primer lugar se explora el papel de Einstein como revolucionario cultural, centránsose en su repercusión en el campo de las letras norteamericanas. Una vez llevada a cabo esta tarea, preliminiar pero indispensable, el artículo rastrea las referencias al científico suizo en el corpus de Vonnegut, así como los numerosos momentos en que postulados teóricos provenientes de la física cuántica sirven para sustentar hilos o motivos argumentales dentro del mismo.

Palabras clave: Narrativa norteamericana, Postmodernismo, Kurt Vonnegut, Albert Einstein.

Abstract: This paper focuses on the deep influence that Albert Einstein and some of his closest disciples and colaborators played on Kurt Vonnegut's narrative. In order to achieve this goal, the study starts by analyzing Einstein's role as a maker of modern culture in general and as a seminal influence on contemporary American novelists in particular. Once this prliminary and indispensable tasks has been fulfilled, I move on to examine Einstein's direct references on Vonnegut's novels as well as how some theories from the field of Quantum Physics become necessary elements in order to sustain some thematic issues or elements within Vonnegut's corpus.

Key words: American narrative, Postmodernism, Kurt Vonnegut, Albert Einstein.

\section{AlBERT EINSTEIN COMO REVOLUCIONARIO CULTURAL}

El 18 de abril de 1955 dejaba de brillar una de las mentes más lúcidas, provocadoras, audaces y verdaderamente revolucionarias de todos 
los tiempos. Albert Einstein, fallecido en Princeton (New Jersey), legaba alrededor de trescientas obras científicas, más de un centenar de textos no eminentemente científicos y un Premio Nobel. Sus publicaciones, conferencias y correspondencia personal albergan un caudal de conocimientos tan vasto e inabarcable como complejo y, en muchas ocasiones, desconcertante. Del mismo modo, sin las aportaciones del teórico de Ulm, el conocimiento que poseemos en la actualidad del universo se vería reducido en gran medida, las leyes de la termodimánica no contarían con la entropía, la producción energética mundial sería diferente, al no contar con la energía nuclear como aliada, el mundo de la informática no habría llegado a avanzar con la rapidez que lo ha hecho, por sólo mencionar algunas de las disciplinas científicas que se han beneficiado de sus investigaciones. En definitiva, no es descabellado aseverar que la muerte de Einstein privaba a la humanidad de una de las mayores mentes de la historia. En contrapartida, el difunto genio dejaba tras de sí un legado lo suficientemente amplio y rico como para continuar abriendo puertas al saber y los avances técnicos y científicos que ha continuado décadas después su muerte. En este sentido, ya en 1950 Leopold Infeld afirmaba que "relativity theory, essentially the work of one man, changed our concepts of space, time, mass, energy, gravitation, and the geometry of the world" (1950: 112).

Si su papel como científico configuraría en gran medida el mundo como se conoce en la actualidad, la huella de Einstein en la historia universal estaba destinada a ser más importante, ya que el científico contaba con el dudoso honor de haber jugado un papel indispensable en la creación de la bomba atómica que desoló las ciudades de Hiroshima y Nagasaki. ${ }^{1}$ Asimismo, a pesar de que Einstein se definió durante toda su vida como un pacifista convencido y de que no tardó en catalogar las misivas en las que abogaba por el uso de armamento nuclear como "[the] great mistake in my life" (Clark 1970: 752), su influencia a la hora de convencer al presidente de Estados Unidos de la conveniencia de apostar por el armamento nuclear jugó un papel decisivo.

Sin lugar a dudas, los bombardeos atómicos tuvieron una relevancia histórica fundamental tanto por la desolación y tragedia humana que causaron de manera inmediata como por las terribles secuelas que aún a

\footnotetext{
${ }^{1}$ Aunque es cierto que Einstein no tuvo participación directa en el desarrollo de la terrible arma nuclear, resulta indudable que sin sus aportaciones al campo de la física, el desarrollo de la energía nuclear se encontraría muy lejos de ser capaz de crear una bomba de ese tipo. Por otra parte, sí que jugó un papel determinante a la hora de promover el lanzamiento de la misma al escribir una serie de cartas al Presidente Roosevelt en las que aconsejaba con la mayor de las vehemencias el incremento en el presupuesto e interés a la hora de desarrollar la bomba atómica.
} 
día de hoy sufren estas poblaciones. No obstante, la bomba atómica iba a jugar un papel mucho más importante en la historia moderna, al mantener al mundo entero sumido en un estado de guerra fría durante medio siglo. Aunque fantasear con qué hubiera ocurrido en caso de que el Enola Gay y el resto de bombarderos que conformaron el terrible escuadrón no hubieran liberado su mortal carga sobre territorio nipón constituya una tarea tan absurda como infructuosa, pocas voces osarían discutir que sin la amenaza de un armagedón casi instantáneo la tensa paz sostenida entre los dos bloques hegemónicos tras la Segunda Guerra Mundial hubiera cedido al peso de unos idearios diametralmente opuestos, pero al servicio de unas ambiciones igualmente desbocadas.

De esta manera, el paso por el mundo de Einstein hizo avanzar las ciencias teóricas y aplicadas de manera exponencial y contribuyó de manera determinante a perfilar el complicado e inestable panorama geopolítico mundial que se había constituido tras la derrota del bando nazi en la Segunda Guerra Mundial. Sin embargo, la importancia del físico para la cultura occidental es aún mayor, al erigirse como una de las mentes filosóficas más brillantes de todos los tiempos. Tras la celebérrima fórmula $\Sigma=m c 2$ se ocultaba una auténtica caja de Pandora filosófica de proporciones indescriptibles. En primer lugar, como Hans Reichenbach apunta "the analysis of knowledge has always been the basic issue of philosophy; and if knowledge in so fundamental a domain as that of space and time is subject to revision, the implications of such criticism will involve the whole of philosophy" (1970: 290).

La física cuántica y la teoría de la relatividad permitían conocer el universo como nunca se había logrado, además de imponer una manera nueva de enfrentarse al mismo, incompatible con concepciones, dogmas y axiomas ancestrales y tradicionalmente aceptados. Desde un prisma cuántico, el universo no debe concebirse de manera lineal, sino más bien como una entidad altamente flexible que se pliega y repliega sobre sí misma, por lo que el concepto de tiempo se problematiza. Este hecho lleva a admitir una nueva dimensión ${ }^{2}$ y la ontológica oposición binaria entre ser/no ser deja de tener sentido. Como Stanley L. Jaki apunta "Einstein's work on relativity was not yet completed when it began to be taken for the scientific proof of the view that everything is relative. Such a view, widely entertained on the popular as well as on the academic level, is now a climate of thought" (1987: 5). En este sentido, el pensamiento de Einstein trasciende el ámbito científico para abrirse paso con una inusitada fuerza en el mundo de la filosofía. Los estudios científicos de Einstein siguen una senda similar

\footnotetext{
${ }^{2}$ Con las excitantes implicaciones que esto conlleva y que abren las puertas a la posibilidad de universos paralelos, agujeros de gusano.
} 
a las teorías de Sigmund Freud o Carl Gustav Jung sobre la mente humana, que enriquecen y hacen avanzar el campo de estudio de la Psicología además de cimentar una visión del ser humano y del mundo alejada de ideas ancestrales y convenciones que no se habían puesto en duda hasta ese momento.

Al respecto de hasta qué punto debe entenderse la figura de Einstein como la de un pensador y revolucionario intelectual a la par que como la de un extraordinario científico, conviene señalar la opinión de Keith R. Burich, quien asegura que "despite his obvious ideological bias, it was Lenin who probably possessed the most perceptive understanding of the moral and philosophical underpinnings of relativity" $(1987: 19){ }^{3}$

De esta manera, es perfectamente coherente definir a Einstein como a un "maker of modern culture", definición que le da Roland N. Stromberg (1991) en un volumen en el que estudia a los cinco pensadores que, desde su punto de vista, han contribuido mejor a cimentar el entendimiento que tenemos del mundo en el siglo XX y albores del XXI. Asimismo, resulta importante señalar la opinión de Arkady Plotnitsky (1994) de que el camino que toman algunos de los filósofos más revolucionarios del siglo XX como Jacques Derrida está ciertamente influenciado por el cambio radical de perspectiva a la hora de entender el universo que propone la física cuántica.

\section{Albert EINStein Y LA Literatura Del Siglo XX}

Tras considerar el monumental impacto que el pensamiento de Einstein tuvo en los más diversos ámbitos de la ciencia, historia, cultura y sociedad del siglo veinte, es lógico pensar que la literatura contemporánea fuera también permeable a las revolucionarias teorías del genio suizo. Al igual que los avances técnicos y el descubrimiento del Nuevo Mundo constituyeron una fuente inagotable de influencias y metáforas para los literatos renacentistas y poetas metafísicos británicos, que la exploración de nuevos mares y tierras ignotas dieron alas a las narraciones de aventuras del siglo XVIII, que los descubrimientos de Tesla y Edison se abrieron paso en la genial novela de Mary Shelley Frankenstein o que las obras de Dickens serían imposibles de entender sin tener en cuenta los avances científicos de la Revolución Industrial y sus consecuencias sociales, la literatura de la segunda mitad del siglo XX refleja ciertos aspectos de la física cuántica y teoría de la relatividad, hasta el punto de verse condicionada por estas corrientes científicas. Como Carol Donley explica: "The poetic responses to relativity appear in two ways: first, writers used Einstein and concepts of relativity as content or subject matter of their

\footnotetext{
${ }^{3}$ Sobre la siempre compleja relación entre la teoría de la relatividad en ciertos aspectos del ideario soviético recomendamos los trabajos de Booker (1994) y Vucinich (2001).
} 
poetry; second, writers invented formal or structural analogues to relativistic space and time" (1987: 119).

Aunque, como se acaba de afirmar, la influencia de los postulados de Einstein se ve reflejada en innumerables movimientos literarios del siglo XX, ésta es especialmente importante en el seno del modernismo y postmodernismo estadounidense. Así, como Stephen R. Mandell apunta, las concepciones científicas de la relatividad y la cuántica y sus connotaciones filosóficas ejercen una influencia notoria en el corpus de determinados poetas modernistas, como William Carlos Williams o Louis Zukovsky (1987: 135).

Si algunos de los miembros más representativos de la poesía modernista abrazan las ideas de Einstein, en el campo de la narrativa las teorías del genio suizo tendrán un eco mayor dentro del campo del postmodernismo. Mientras que la novela modernista había buscado huir de la narrativa decimonónica adentrándose en las profundidades de la mente humana, apoyándose en Freud y el psicoanálisis, numerosos novelistas postmodernos verán en las ideas de Einstein una manera equivalente de esquivar el realismo literario porque, como Christopher Norris señala, la misma esencia de los postulados de Einstein "deny the knowability and hence the very existence of objective or verification-transcendent truths" (2000: 40) y, por lo tanto, hace imposible una aproximación realista a ningún fenómeno que tenga lugar en el universo. De esta forma, un número significativo de escritores del siglo XX descubrirán en las ideas de Einstein un correlato perfecto a su propia concepción del mundo, puesto que como Robert Hauptman e Irving Hauptman explican en su artículo "The Circuitous Path: Albert Einstein and the Epistemology of Fiction":

The zeitgeist and the general inferences drawn from Einstein's work come to bear most seminally on a group of philosophically oriented novelists conveniently termed absurdists, authors who believe that man is, in Heidegger's phrase, "thrown" into a world devoid of absolutes, order, and meaningfulness. The world that these novelists depict is one in which external meaning is elusive, metaphysical underpinnings are questioned, and knowledge is ephemeral (1987: 125).

Aunque en esta cita los autores se refieran a novelistas del absurdo y en el texto de su artículo traten específicamente de creadores como Franz Kafka, Jean Paul Sartre o Albert Camus, esta apreciación puede hacerse extensiva a la narrativa de un gran número de narradores postmodernos, como por ejemplo John Barth, Donald Barthelme o, más recientemente Chuck Palahniuk o Douglas Coupland, que comparten una visión del mundo como caótico y resistente a cualquier intento humano de reducirlo a unas leyes lógicas de cognoscibilidad. 
Además de servir como sustento para el sentido de la existencia universal que mantienen algunos de los más relevantes narradores del siglo veinte, las teorías de Einstein permiten articular los hilos argumentales de innumerables novelas del período. Un postulado científico que abre la puerta a un universo que se pliega sobre sí mismo y alberga agujeros de gusano que abren la puerta al viaje interdimensional (por no hablar de la naturaleza no lineal del tiempo) excitará la imaginación de incontables escritores del momento, muy especialmente dentro del género de la ciencia ficción. ${ }^{4}$

Se ha tratado hasta el momento la enorme repercusión que tienen las teorías expuestas por Einstein en el seno de la comunidad científica y en el ambiente político y cultural del siglo veinte. Igualmente se ha expuesto de manera somera cómo la literatura norteamericana del momento las aceptó con auténtico entusiasmo. A continuación se analizará cómo la figura de Einstein y sus descubrimientos cobran una importancia capital en el corpus literario del autor objeto de estudio en el presente trabajo.

\section{LA SOMBRA DE EINSTEIN EN LA NARRATIVA DE KURT VONNEGUT}

Es relevante comenzar dicha tarea apuntando que a Vonnegut cabe presumirle un conocimiento de las principales teorías de Einstein y la física cuántica más que superficial, aunque no demasiado profundo. En primer lugar, se hace pertinente señalar que en 1952 (fecha en que el escritor publica su ópera prima) Einstein había traspasado ya los entornos académicos y profesionales para convertirse en un personaje célebre y de sobra conocido por la sociedad norteamericana. Así pues, resulta difícil concebir que un ciudadano de clase media y educación universitaria como lo era Vonnegut pudiera estar al margen de, al menos, los postulados más básicos de la recién nacida física cuántica y teoría de la relatividad. Es más, prestando atención a la biografía del autor es fácil comprender que, a pesar de que éste no haya hecho nunca mención expresa al respecto, Vonnegut conocía las ideas de Einstein de forma muy superior al del común de ciudadanos de clase media norteamericanos. En concreto, es impensable que Vonnegut pasara tres años en Cornell estudiando Bioquímica sin entrar en contacto con las ideas vertidas por la nueva física. ${ }^{5}$ Además, el propio Vonnegut ha manifestado en numerosas entrevistas que su trabajo en GE le

\footnotetext{
${ }^{4}$ Por supuesto, esta influencia no es exclusiva del género de la ciencia ficción, ya que, por ejemplo, determinados pasajes de Thomas Pynchon serían ininteligibles sin tener en cuenta determinados conceptos provenientes de la física cuántica y la termodinámica avanzada.

${ }^{5}$ No podemos olvidar que en 1943 , fecha en que Vonnegut se desvincula de Cornell, hacía 38 años que Albert Einstein había introducido la teoría de la relatividad en su célebre "On the Electrodynamics of Moving Bodies" y 22 años desde que había sido galardonado con el Premio Nobel de Física.
} 
permitió estar en contacto directo y diario con alguno de los científicos más prominentes del momento, al mismo tiempo que le obligaba a estar al día en lo concerniente a los últimos descubrimientos y desarrollos científicos. ${ }^{6}$

Pese a que Vonnegut no haya hecho menciones explícitas a la figura de Einstein o la teoría de la relatividad ni en sus entrevistas ni en sus numerosos discursos públicos, las ocasiones en que estos elementos se presentan en su corpus literario son numerosas. Así, a este personaje se refiere en un total de siete novelas, en dos de sus tres colecciones de relatos breves y en la obra God Bless You, Dr. Kevorkian. ${ }^{7} \mathrm{Si}$ el hecho de que el científico aparezca citado en casi el cincuenta por ciento de los libros del autor resulta ya ciertamente sintomático para entender hasta qué punto está presente la figura de Einstein en Vonnegut mientras éste compone sus textos literarios, es interesante comparar esta extraordinaria profusión de referencias con las ocasiones en que personajes como Franklin Delano Roosevelt o Charles Darwin (a los que el autor se ha referido en innumerables ocasiones, principalmente en sus comparecencias públicas, afirmando que sin estos personajes históricos él nunca habría llegado a ser la persona en que finalmente se convirtió). En concreto, frente a las 10 referencias a Einstein en el corpus del escritor se dan 9 a Roosevelt y 8 a Darwin. De esta manera, las únicas personas que se ven reflejadas en la narrativa de Vonnegut con mayor frecuencia que el genio suizo son exclusivamente los miembros de su propia familia que, de una u otra manera, se abren paso a lo largo de todas y cada una de sus creaciones literarias.

Aunque en algunos de los textos de Vonnegut las referencias al científico suizo son francamente irrelevantes para la trama argumental principal, en ciertas novelas las referencias a Einstein ponen en evidencia la gran influencia que ejercieron éste y sus teorías en el novelista de

\footnotetext{
${ }^{6}$ Como ejemplo de hasta qué punto GE daba cobijo a alguna de las mentes científicas más lúcidas del momento nos gustaría señalar que uno de los jefes de Vonnegut fue el Dr. Irving Langmuir, el primer físico que conseguía un Premio Nobel (1932) desarrollando sus investigaciones en el entorno profesional y no en el ámbito universitario.

${ }^{7}$ En las novelas Jailbird y Timequake se menciona a Albert Einstein en siete y cinco ocasiones respectivamente.

${ }^{8}$ El listado completo de obras en que aparecen los personajes históricos referidos es el siguiente:

Albert Einstein aparece en: The Sirens of Titan; Breakfast of Champions; Jailbird; Slapstick, or Lonesome no More!; Bluebeard; Timequake; Hocus Pocus; Welcome to the Monkey House; Bagombo Snuff Box y God Bless You, Dr. Kevorkian .Franklin Delano Roosvelt aparece en: Mother Night; Cat's Cradle; Jailbird; Slapstick, or Lonesome no More!; Deadeye Dick; Hocus Pocus; Bagombo Snuff Box; Fates Worse than Death y God Bless You, Mr. Rosewater .Charles Darwin, por su parte, es mencionado en los siguientes libros: Slaughterhouse-Five; Jailbird; Slapstick, or Lonesome no More!; Galápagos; Timequake; Hocus Pocus; God Bless You, Dr. Kevorkian y Fates Worse than Death.
} 
Indianápolis. ${ }^{9}$ Como ejemplo de estas obras Breakfast of Champions y Slapstick son significativas, porque en ellas Einstein se presenta como la quinta esencia del talento humano y God Bless You, Dr. Kevorkian, donde Vonnegut entrevista a Sir Isaac Newton, quien asegura odiarse a sí mismo por no haber dado con la teoría de la relatividad antes que Einstein. Más interesante que los ejemplos anteriores es, sin duda, la novela Jailbird, donde Vonnegut afirma que con su teoría de la relatividad Einstein había descubierto la rueda sino que el novelista va un paso más allá, al convertir al científico en personaje de la novela. ${ }^{10}$ Este hecho es especialmente significativo si se tiene en cuenta que en el corpus vonnegutiano el único personaje histórico que se convierte en personaje de ficción, además de Einstein, es Ernest Hemingway, que protagoniza la obra de teatro Happy Birthday, Wanda June (1971).

\section{LA FÍSICA CUÁNTICA COMO EXCUSA ARGUMENTAL}

Gran parte de los estudios académicos (y los editores de las primeras novelas del autor) catalogaron una parte significativa de la producción literaria de Vonnegut, muy a su pesar, como perteneciente al género de la ciencia ficción. La frecuencia con que el autor acudía a una serie de argumentos y elementos temáticos que dependían de unos avances tecnológicos impensables en el momento en que los textos vieron la luz, sin duda contribuyeron a este etiquetado.

Ya desde su ópera prima, Vonnegut apostaba por situar la acción en un futuro indeterminado, aunque cercano, en el que los avances tecnológicos habían evolucionado hasta tal punto que la labor de los seres humanos se veía amenazada como mano de obra no cualificada. Sin embargo, los inventos y avances mostrados en Player Piano recuerdan a la Revolución Industrial y nos hacen pensar en la introducción de los modernos electrodomésticos en los hogares norteamericanos más que transportar al lector a un futuro remoto cuyas posibilidades científicas

${ }^{9}$ Como veremos en capítulos posteriores, la afirmación que acabamos de realizar se hace asimismo extensiva a los personajes históricos de Franklin Delano Roosevelt y Charles Darwin.

${ }^{10}$ Para ser precisos, Einstein se convierte en personaje de un relato que inventa el personaje de Kilgore Trout dentro de la novela Jailbird. En dicho relato, titulado "Dormido en el cambio de vía", se presenta al espectro del genio suizo que acaba de fallecer y cruza las puertas del cielo. En él se dice que al final de su vida Einstein "era un veterano de innumerables cruces de frontera por entonces. Siempre le habían hecho preguntas absurdas, le habían obligado a hacer hueras promesas y a firmar documentos intrascendentes". Asimismo, es especialmente importante señalar que en este relato Vonnegut muestra a Einstein como un genio científico además de como un pensador genial y revolucionario cuya mentalidad crítica y capacidad de generar cuestiones en el resto de seres humanos son tan acusadas que hacen enfurecer al propio Dios. 
resultan impensables para nuestras mentes contemporáneas. En otras palabras, las máquinas de Player Piano eran altamente sofisticadas, e imposibles en la década de los cincuenta, pero no dejaban de ser máquinas, es decir, elementos de uso cotidiano en la mayoría de hogares del momento. De esta manera, para seguir la admonición de Coleridge de suspender voluntariamente su incredulidad en pos de disfrutar de la obra literaria, el lector de Player Piano simplemente tenía que aceptar como posible un nivel de desarrollo tecnológico (de carácter mecánico y diseño un tanto rudimentario) muy superior al de su propio contexto sociocultural, pero en ningún momento inconcebible para él.

The Sirens of Titan (1959), sin embargo, basaba su argumento principal en la existencia de un elemento en el universo nuevo y diferente a todas y cada una de las concepciones que se habían venido manteniendo del cosmos a lo largo de la historia de la humanidad, tanto desde una perspectiva científica como moral o incluso ontológica. Este hecho se debe a que la premisa argumental de la novela depende por completo de la posibilidad de un fenómeno llamado "chrono-synclastic infundibulum", cuya mera posibilidad desafiaba las propias bases de la concepción que se tenía del universo antes de la irrupción en la esfera intelectual y científica de la teoría de la relatividad. Este extraño fenómeno se define en la novela de la siguiente forma:

Chrono (kroh-no) means time. Synclastic (sin-class-tick) means curved toward the same side in all directions, like the skin of an orange. Infundibulum (in-fun-dib-u-lum) is what the ancient Romans like Julius Caesar and Nero called a funnel. If you don't know what a funnel is, get Mommy to show you one (11).

Si la explicación etimológica del fenómeno es desconcertante, las consecuencias de adentrarse en uno de estos chrono-synclastic infundibulum resultan terroríficas:

Winston Niles Rumfoord had run his private space ship right into the heart of an uncharted chrono-synclastic infundibulum two days out of Mars. Only his dog had been along. Now Winston Niles Rumfoord and his dog Kazak existed as wave phenomena - apparently pulsing in a distorted spiral with its origin in the Sun and its terminal in Betelgeuse (ibid).

Puesto que el protagonista de la novela y su perro son ahora "wave phenomena", no poseen existencia física como se conoce en la tercera dimensión ni están sujetos a las reglas temporales que rigen en la esfera de existencia humana. Así, en The Sirens of Titan, Vonnegut juega con una idea que había venido siendo la base de bastantes narrativas de ciencia ficción: la del viaje en el tiempo. Pero la lleva a un extremo que sería 
imposible de conceptualizar sin utilizar los postulados ofrecidos por la teoría de la relatividad. Por fantástica e irrealizable que pudiera ser, la posibilidad de realizar un viaje a través del tiempo, no resultaba inconceptualizable para el lector de los años 50. En primer lugar, como David Lewis explica, viajar en el tiempo conlleva una serie de implicaciones:

What is time travel? Inevitably, it involves a discrepancy between time and time. Any traveler departs and then arrives at his destination; the time elapsed from departure to arrival (positive, or perhaps zero) is the duration of the journey. But if he is a time traveler, the separation in time between departure and arrival does not equal the duration of his journey. He departs; he travels for an hour, let us say; then he arrives. The time he reaches is not the time one hour after his departure. It is later, if he has traveled toward the future; earlier, if he has traveled toward the past (1993: 134).

En otras palabras, desplazarse en el tiempo constituye una imposibilidad, puesto que rompe con las más elementales reglas naturales tal y como las conocemos a día de hoy, pero a fin de cuentas no supone sino viajar de un momento a otro en el tiempo. Así pues, el lector del siglo $\mathrm{XX}$ probablemente no creyera en los viajes temporales, pero tampoco encontraría grandes obstáculos a la hora de suspender su incredulidad, siempre de manera voluntaria y temporal, a la hora de encontrarse con uno de estos desplazamientos temporales en una obra literaria. Por otra parte, y empleando la terminología del filósofo suizo Carl Jung, la idea del viaje intertemporal puede incluso ser considerada como un arquetipo universal presente desde tiempos inmemoriales en el imaginario colectivo. La posibilidad del desplazamiento temporal como arquetipo universal no debe sorprendernos, puesto que, con la providencial espada de Damocles de la propia muerte que pende sobre los seres humanos, el deseo de conocer qué ocurrirá siglos después de que nos hayamos convertido en polvo resulta una fantasía de lo más prometedora.

Tratar de esbozar aquí una lista de las obras literarias más significativas en que se ha usado el recurso del viaje temporal como parte del argumento de las mismas es un objetivo tan inabarcable como ajeno al objeto de nuestro estudio, por lo que nos limitaremos a señalar que en 1773 Samuel Madden publica la que tiene el honor de ser considerada como primera novela moderna que incluye el viaje temporal: Memoirs of the Twentieth Century. A partir de esa fecha, las obras las en que se incluye esta temática se multiplican hasta llegar a los albores del siglo XX, donde H.G. Wells cimenta las convenciones de la narrativa de viajes intertemporales, subgénero muy popular dentro de la ciencia ficción, con The Chronic Argonauts (1888) y muy especialmente con la conocida The 
Time Machine (1895). ${ }^{11}$ Aunque la temática del viaje a través del tiempo suele presentarse de manera muy recurrente dentro del género de la ciencia ficción, es fundamental resaltar que desde sus mismos orígenes, también ha sido empleada por autores que poco o nada tienen que ver con este tipo de literatura. Por citar tan sólo dos de los ejemplos más conocidos referenciamos las novelas de Mark Twain, A Connecticut Yankee in King Arthur's Court (1889) y The Star Rover de Jack London (1915).

Por tanto, las fantasías sobre los viajes temporales eran de sobra conocidas para el lector en el año en que The Sirens of Titan vio la luz, puesto que las letras universales llevaban jugando con ella durante siglos hasta el punto en que, como Bud Foote (1991) apunta, en el siglo veinte se había convertido en un auténtico topoi dentro de determinados géneros literarios. De esta manera, el hecho de que Winston Niles Rumfoord y su perro Kazak puedan desplazarse a través del tiempo no suponía al lector de The Sirens of Titan un elemento novedoso ni sorprendente. Sin embargo, la naturaleza del "chrono-synclastic infundibulum" que idea Vonnegut para la novela, y que sería inconcebible sin las teorías expresadas por Einstein, sí que resulta revolucionaria.

En primer lugar, el "agujero" por el que el protagonista de la novela y su mascota se adentran no hace de ellos seres capaces de viajar a través del tiempo, sino que los convierte en "wave phenomena". Es decir, en seres desprovistos de la estructura orgánica humana. Al ostentar esta condición ni Rules ni Kazak poseen un cuerpo como tal, sino que sus moléculas se encuentran desestructuradas. Además, se hallan presentes en diversos planos espaciales de manera simultánea. Esto conduce directamente al siguiente punto verdaderamente original de la obra de Vonnegut: al plantear esta posibilidad, Vonnegut pide al lector que crea no en un viaje en el tiempo sino en la posibilidad del don de la ubicuidad. Sin embargo, la cuestión se complicará aún más, ya que no es que los personajes se encuentren en diversos lugares al mismo tiempo sino que no están en ninguno de ellos al ser incorpóreos y poder materializarse exclusivamente cada cierto tiempo, cuando acontece un determinado fenómeno estelar. En definitiva, para suspender temporalmente su incredulidad y adentrarse en la trama de The Sirens of Titan el lector se veía forzado a aceptar tres premisas que cuestionaban la naturaleza misma de la realidad como se había venido entendiendo durante milenios. En primer lugar, el universo no es lineal, sino que se pliega sobre sí mismo y, por lo tanto, da cabida a entidades astrales como los agujeros negros. En segundo lugar, la

11 Como nota marginal pero interesante, señalaremos que la primera obra literaria en que aparece una máquina del tiempo corresponde a un autor español, y se trata de $E /$ Anacronopete (1887), de Enrique Gaspar y Rimbau. 
posibilidad de existir de forma paralela en dos lugares al mismo tiempo es un hecho que se produce constantemente en el universo aunque el ojo humano no sea capaz de percibirla. Por último, el don de la ubicuidad deja de ser exclusivo de Dios, quien debe compartir semejante privilegio con diversas partículas. Así pues, aceptar la posibilidad de un "chrono-synclastic infundibulum" requería derribar tres hechos tradiconalmente aceptados, relacionados tanto con la estructura física del universo como con su dimensión moral.

De las afirmaciones expresadas con anterioridad se derivan nuevas consecuencias, que dinamitan los cimientos de la ciencia tradicional y, por lo tanto, de la manera de entender el universo y la vida. Si los átomos pueden separarse y no están permanentemente cohesionados y existe, además, la posibilidad de existencias simultáneas en lugares diversos, los tradicionales conceptos de espacio y tiempo pierden todo su sentido. Como pasamos a mostrar a continuación, el concepto de "chrono-synclastic infundibulum" que Vonnegut plantea en su segunda novela es sin duda fantástico, pero éste se basa en los descubrimientos y teorías realizadas por Einstein y alguno de sus más aventajados discípulos.

En primer lugar, analizaremos la concepción atómica de la naturaleza. La idea de que la materia estaba formada por elementos muy pequeños $y$, por lo tanto, inaprensibles para el ojo mortal se había venido forjando con los obvios vaivenes intelectuales y científicos que implicaban los diversos cambios de era, concepción política y teológica del mundo. Pero estas ideas bebían directa y casi exclusivamente de las fuentes de la filosofía. Sin embargo, a partir de finales del siglo XIX, el avance tecnológico y científico permitiría demostrar la naturaleza atómica de la materia. Ya en 1827 Robert Brown se apoyó en un microscopio para validar las teorías expresadas en 1803 por John Dalton. No será, sin embargo, hasta 1909 cuando Ernest Rutherford consiguiera hacer posible lo que ya había propuesto J. J. Thomson en 1897: hacer "visibles" ( $\mathrm{y}$, por lo tanto, materia de investigación empírica) a los átomos bombardeándolos con iones de helio de manera que ciertos átomos produjeran un reflejo mucho mayor que ellos mismos al alcance de los materiales de observación científica del momento.

El descubrimiento más determinante lo realizaría Erwin Schrödinger en 1926. Schrödinger, un convencido seguidor de la teoría de la relatividad y amigo íntimo de Einstein que tenía por costumbre mostrar sus hallazgos al genio suizo vía epistolar antes de presentarlos a la comunidad científica, afirmó que las partículas atómicas no se comportaban como elementos fijos en un espacio determinado sino que podían entenderse como ondas en movimiento continuo y permanente. Vonnegut expone que el resultado de adentrarse en un "chrono-synclastic infundibulum" es el de convertirse en 
"wave phenomena", esto es, el novelista lleva la teoría atómica a sus últimas consecuencias (que no son, desde luego y como resulta evidente, posibles fuera del terreno de la ficción). ${ }^{12}$ De esta manera, el novelista convierte a los protagonistas de la novela no ya en viajeros en el tiempo sino en algo radicalmente diferente. Para llevar a cabo este propósito se vale no de una imaginación desorbitada sino de los postulados científicos de Einstein y Schrödinger.

Para posibilitar que sus personajes se conviertan en fenómenos de ondas y esquiven las leyes físicas que imperan entre los elementos complejos para comportarse como átomos, Vonnegut recurre de nuevo a teorías provenientes de la física cuántica, en este caso a los popularmente conocidos como agujeros de gusano. ${ }^{13}$ En 1935 Einstein firmó, junto con Nathan Rosen, un artículo que ahondaba en la naturaleza del universo y su estructura. ${ }^{14}$ Aparecido en Physical Review, "The Particle Problem in the General Theory of Relativity" afirmaba que si el universo no se extiende de manera lineal como se había venido considerando sino que mantiene una compleja estructura flexible en la que se pliega y despliega sobre sí mismo en innumerables ocasiones, sería posible pasar de un punto a otro del universo atravesando dichos pliegues. Explicando esta teoría de acuerdo con el nombre que le aplica Wheeler, se debe imaginar un gusano posado sobre la superficie de una manzana. Si dicho gusano debiera transitar el perímetro externo de la manzana se vería obligado a recorrer un camino mucho más largo que si optase por tomar un hipotético hueco transversal que llevara de la parte superior de la fruta a la inferior. De esta manera, al igual que el nematodo toma un atajo al atravesar la manzana en lugar de rodear su perímetro, los agujeros de gusano supondrían un atajo entre espacio y tiempo al desplazarse entre puntos distantes del universo.

Aunque no era el interés de Einstein o Rosen el de sugerir la posibilidad de la existencia de agujeros de gusano que permitieran los desplazamientos físicos a través del universo, dicha posibilidad queda desde luego implícita en el artículo donde se expresa la esencia de los

\footnotetext{
${ }^{12}$ A pesar de que esta afirmación pueda resultar evidente, consideramos pertinente hacerla, debido a la proliferación en los últimos años de pseudo-científicos adeptos a ciertos programas de televisión que se han empeñado en esgrimir las teorías de Einstein y Schrödinger para justificar la posibilidad de los desplazamientos temporales tal y como los entiende la ciencia ficción.

${ }^{13}$ Nomenclatura acuñada por John Wheeler, que también crea el conocido término "agujero negro".

${ }^{14}$ Aunque son Einstein y Rosen los que estudian con mayor acierto este fenómeno del cosmos, cabe reconocerle a Ludwig Flamm el haber dedicado por primera vez atención a estos agujeros en el espacio, siguiendo también las teorías de Einstein, en 1916.
} 
agujeros de gusano. ${ }^{15}$ En este sentido es importante introducir las teorías de John Wheeler, quien en 1955 traza una clara distinción entre dos tipos de agujeros de gusano: los Lorentzian y los Euclidean. ${ }^{16}$ Sin abordar en profundidad el pensamiento físico teórico de Wheeler, baste decir que los agujeros de tipo Euclidean no serían atravesables, mientras que los Lorentzian, por el contrario, ofrecerían un puente entre diferentes puntos del universo, o incluso entre universos diferentes, permitiendo (siempre en el plano teórico) su uso para desplazamientos cósmicos.

Como se desprende de las teorías de Einstein y Rosen primero y Wheeler después, el "chrono-synclastic infundibulum" de The Sirens of Titan podría catalogarse como un hipotético puente de Einstein-Rosen de tipo Laurentzian.

En obras posteriores el novelista tiene presentes de nuevo las ideas de Einstein y alguno de sus discípulos, aunque de manera mucho más velada. Así, en Slapstick las premisas argumentales son fantásticas, pero esta vez no están relacionadas con los postulados de Einstein. En primer lugar, la novela tiene lugar en un momento en que la tierra experimenta unas extravañas fluctuaciones de gravedad que provocan que los cuerpos sobre la Tierra se vuelvan casi ingrávidos en determinados momentos. El segundo postulado fantástico en el que se apoya la trama de la novela consiste en que, a través de una técnica sofisticada, los chinos han sido por fin capaces de hacerse con el poder mundial. Esta nación se hace con el control planetario mediante una técnica de miniaturización de sus soldados que, de esta manera, son capaces de penetrar en los cuerpos de ciudadanos de otras naciones haciéndolos enfermar. En estos argumentos no se puede encontrar relación entre los fenómenos descritos y los de la física cuántica.

Sin embargo, Vonnegut indica de forma clara en Slapstick que los postulados fantásticos de la novela tienen su punto de partida no sólo en los descubrimientos de Einstein sino en cómo éstos modificaron el entendimiento del universo. En primer lugar, como ya se apuntó con anterioridad, en Slapstick hay una referencia textual a Einstein en la que se le cataloga como uno de los mayores genios de la historia de la humanidad. Para Vonnegut, Einstein es un genio por su capacidad para descubrir e

\footnotetext{
${ }^{15}$ En cualquier caso siempre teóricos o hipotéticos, puesto que no podemos olvidar que los agujeros de gusano no dejan de ser agujeros negros y, por lo tanto, el mero hecho de adentrarse en uno de ellos supondría para el ser humano la desaparición instantánea puesto que la extrema densidad de los agujeros negros y tendencia expansiva absorben absolutamente toda partícula que se aproxima a ellos, incluyendo la luz.

${ }_{16}$ El autor revisa esta teoría con mayor detenimiento y detalle en una obra posterior que se convertiría en seminal para los interesados en el fascinante campo de los agujeros negros y sus diversas variantes en el trabajo Geometrodynamics (1962).
} 
identificar nuevas posibilidades, al igual que le ocurre a los protagonistas de la novela: "We supposed that it might even be abnormal on earth for gravity to be stable for long periods of time. We predicted that at any moment gravity might become as capricious as winds and heat and cold, as blizzards and rainstorms again" (37).

La segunda referencia indirecta a Einstein y su grupo de seguidores ocurre cuando el narrador de la novela reflexiona sobre cómo los chinos han sido capaces de alcanzar un nivel de desarrollo tecnológico tan avanzado y lo expresa en los siguientes términos: "the Chinese had begun to combine those synthetic minds into intellects so flabbergasting that the Universe itself seemed to be saying to them, 'I await your instructions. You can be anything you want to be. I will be anything you want me to be'" (68). Vonnegut se esfuerza por hacer aún más patente que las mentes brillantes que inspiran los genios de su novela no son los seguidores de Einstein y sus postulados: "The Chinesse got the idea from the American and European scientists who put their heads together during the Second World War, with the singlenminded intention of creating an Atomic Bomb" (68).

En su última novela, Timequake (1998), Vonnegut volverá a visitar la figura de Einstein con gran interés. En Timequake se juega con la idea de una ruptura en el equilibrio espacio-tiempo que provoca que los seres humanos se vean inmersos en un bucle que les hace vivir una y otra vez el mismo momento de sus vidas de manera perpetua: "The premise of Timequake One was that a sudden glitch in the space-time continuum, made everybody and everything do exactly what they'd done during a past decade (xiii)".

La influencia del científico Albert Enstein en la novela queda confirmada por las numerosas referencias al mismo, así como por el hecho de que en el epílogo que pone fin a Timequake, Vonnegut ofrece tres citas procedentes del mismo, siendo la más relevante la que se cita a continuación: "Physcial concepts are free creations of the human mind, and are not, however it may seem, uniquely determined by the external world" (215).

Para concluir, la teoría de la relatividad y la nueva concepción que se abría del cosmos tomando en consideración los postulados y teorías de Einstein cobran importancia en las novelas analizadas. No obstante, el verdadero interés de Vonnegut en estas obras está muy alejado de los avances científicos en sí mismos. Hace uso de ellos sólo para crear un hilo argumental que le permita la posibilidad de explorar las cuestiones que verdaderamente le atraen (obsesionan, nos aventuraríamos a decir): cuestiones de índole filosófica y social, no científica. Así, aunque para sustentar el argumento de The Sirens of Titan Vonnegut se apoye en la teoría de la relatividad y cree un "chrono-synclastic infundibulum" similar a 
los puentes de Einstein-Rosen, el verdadero objeto de estudio que se presenta en la novela se hace patente desde su mismo comienzo: "Everyone now knows how to find the meaning of life within himself. But mankind wasn't always so lucky. Less than a century ago men and women did not have easy access to the puzzle boxes within them" (7). The Sirens juega con determinados postulados científicos provenientes de la física teórica más avanzada. Sin embargo, desde las líneas que abren la novela el escritor explicita que el objeto de su interés es el mismo que ha venido obsesionando a filósofos, artistas, pensadores y al común de los mortales desde tiempos inmemoriales: tratan de alcanzar una respuesta para una pregunta tan omnipresente como irresoluble: cuál es el verdadero sentido de nuestra existencia.

Slapstick se vale de las teorías científicas para analizar la soledad que sufre el hombre en la sociedad contemporánea y las posibles soluciones a ésta. Como el propio autor expone: "It is about desolated cities and spiritual cannibalism and incest and loneliness and lovelessness and death, and so on" (13).

Por último, Timequake emplea el argumento de la ruptura en el continuo espacio-temporal como premisa para una obra que termina siendo una extraña mezcla de ficción, elementos autobiográficos y divagaciones de todo tipo en la que la ciencia en sí cobra poca o ninguna importancia, ya que estos elementos no atraen la atención del escritor sumido en un estado de pesimismo como el que se refleja en una novela en la que se asegura lo siguiente: "It appears to me that the most highly evolved Earthling creatures find being alive embarrassing or much worse" (1) y cuyo primer capítulo concluye aseverando que "being alive is a crock of shit" (3).

REFERENCIAS BIBLIOGRÁFICAS

BOOKER, Kevin, The Dystopian Impulse in Modern Literature: Fiction as Social Criticism. Westport, Conn.: Greenwood Press, 1994

CLARK, Robert, Einstein; the Life and Times. New York: Avon Books, 1970.

DONLEY, Carol, "Springtime of the Mind: Poetic Responses to Einstein and

Relativity". En: RYAN, D. (ed.), Einstein and the Humanities. New York:

Greenwood Press, 1987. pp. 119-25.

EVERDELL, William, The First Moderns: Profiles in the Origins of TwentiethCentury Thought. Chicago: University of Chicago Press, 1997.

Flamm, Ludwig., "Comments on Einstein's Theory of Gravity". En: Physikalische Zeitschrift 17, 1916, p. 448.

GASPAR Y RIMBAU, Enrique, El Anacronópete. Barcelona: Minotauro, 2005.

HAUPTMAN, Robert, "The Circuitous Path: Albert Einstein and the Epistemology of Fiction". En: RYAN, D. (ed.), Einstein and the Humanities. New York: Greenwood Press, 1987. pp. 125-35. 
INFELD, Leopold, Albert Einstein, His Work and Its Influence on Our World. New York: Charles Scribner's Sons, 1950.

JAKi, Stanley, "The Absolute beneath the Relative: Reflections on Einstein's

Theories". En: RYAN, D. (ed.), Einstein and the Humanities. New York: Greenwood Press, 1987. pp. 5-19.

LEWIS, C.S., "On Science Fiction". En: Knight, D. (ed.), Turning Points: Essays on the Art of Science Fiction. New York: Harper \& Row, Publishers, 1977. 119-31.

. "The Paradoxes of Time Travel". En: Le Poidevin, R. (ed.), The Philosophy of Time. New York: Oxford University Press, 1993. 13449.

LONDON, Jack, The Star Rover. Amherst, N.Y.: Prometheus Books, 1999.

MAdDEn, Samuel, Memoirs of the Twentieth Century, Being Original Letters

of State under George the Sixth. New York: Garland Pub., 1972.

MANDELL, Stephen, "A Search for Form: Einstein and the Poetry of Louis Zukofsky and William Carlos Williams" En: RYAN, D. (ed.), Einstein and the Humanities. New York: Greenwood Press, 1987. pp. 135-43.

NoRRIS, Christopher, Quantum Theory and the Flight from Realism: Philosophical Responses to Quantum Mechanics. New York: Routledge, 2000.

PlotNITSKY, Arkady, Complementarity: Anti-Epistemology after Bohr and Derrida. Durham, NC: Duke University Press, 1994

REICHENBACH, Hans, "The Philosophical Significance of the Theory of

Relativity”. En: ScHILPP, P. (ed.), Albert Einstein: Philosopher-Scientist. La

Salle, II: Open Court, 1970. pp. 269-87.

ROSEN, Nathan, "The Particle Problem in the General Theory of Relativity".

En: Physical Review 48, 1935, pp. 73-77.

RUTHERFORD, Ernest, "The Electrical Structure of Matter". En: Science 58, 1926, pp. 209-221.

SCHRÖDINGER, Erwin, "Die Gegenwärtige Situation in Der Quantenmechanik". En: Die Naturwissenschaften 21, 1935, pp. 84449.

Stromberg, Roland, Makers of Modern Culture: Five Twentieth-Century Thinkers. Arlington Heights, IL.: Harlan Davidson, 1991.

TwaIN, Mark, A Connecticut Yankee in King Arthur's Court. New York: Norton, 1982.

VonNEGUT, Kurt, Player Piano. New York: Charles Scribner's sons, 1952. , The Sirens of Titan. New York: Delacorte Press, 1959. Welcome to the Monkey House; a Collection of Short Works. New York: Delacorte Press, 1968. Slaughterhouse-Five; or, the Children's Crusade, a Duty-Dance with Death. New York: Delacorte Press, 1969. 
, Happy Birthday, Wanda June; a Play. New York: Delacorte Press, 1971.

, Slapstick : Or Lonesome No More! London: J. Cape, 1976.

Jailbird : A Novel. New York: Delacorte Press/Seymour Lawrence, 1979.

, Deadeye Dick. New York, N.Y.: Delacorte Press/Seymour Lawrence, 1982.

, Galápagos. New York: Delacorte Press/Seymour Lawrence, 1985.

Bluebeard. New York: Delacorte/PressSeymour Lawrence, 1987.

, Hocus Pocus. New York: Putnam's, 1990.

- Fates Worse Than Death : An Autobiographical Collage of the 1980s. New York: G.P. Putnam's, 1991.

, Timequake. New York: G.P. Putnam's, 1997.

, God Bless You, Dr. Kevorkian. New York: Seven Stories Press, 1999.

Bagombo Snuff Box: Uncollected Short Fiction. New York: G.P. Putnam's Sons, 1999.

VuCINICH, Alexander, Einstein and Soviet Ideology. Stanford: Stanford University Press, 2001.

WelLs, H.G., The Chronic Argonauts. London: Kessinger Publishing, 2004. The Time Machine. Philadelphia: Copper Penny Press, 2008.

WHEELER, John, Geometrodynamics. New York: Academic Press, 1962. 